The Astrophysical Journal, 508:435-439, 1998 December 1

(C) 1998. The American Astronomical Society. All rights reserved. Printed in U.S.A.

\title{
SECONDARY COSMIC MICROWAVE BACKGROUND ANISOTROPIES IN A UNIVERSE REIONIZED IN PATCHES
}

\author{
ANDREI GRUZINOV AND WAYNE Hu ${ }^{1}$ \\ Institute for Advanced Study, School of Natural Sciences, Princeton, NJ 08540 \\ Received 1998 March 17; accepted 1998 July 2
}

\begin{abstract}
In a universe reionized in patches, the Doppler effect from Thomson scattering off free electrons generates secondary cosmic microwave background (CMB) anisotropies. For a simple model with small patches and late reionization, we analytically calculate the anisotropy power spectrum. Patchy reionization can, in principle, be the main source of anisotropies on arcminute scales. On larger angular scales, its contribution to the CMB power spectrum is a small fraction of the primary signal and is only barely detectable in the power spectrum with even an ideal, i.e., cosmic variance limited, experiment and an extreme model of reionization. Consequently, patchy reionization is unlikely to affect cosmological parameter estimation from the acoustic peaks in the CMB. Its detection on small angles would help determine the ionization history of the universe, in particular, the typical size of the ionized region and the duration of the reionization process.
\end{abstract}

Subject headings: cosmic microwave background - cosmology: theory — intergalactic medium large-scale structure of universe

\section{INTRODUCTION}

It is widely believed that the cosmic microwave background (CMB) will become the premier laboratory for the study of the early universe and classical cosmology. This belief relies on the high precision of the upcoming MAP $^{2}$ and Planck Surveyor ${ }^{3}$ satellite missions and the high accuracy of theoretical predictions of CMB anisotropies given a definite model for structure formation ( $\mathrm{Hu}$ et al. 1995). To realize the potential of the $\mathrm{CMB}$, aspects of structure formation affecting anisotropies at only the percent level in power must be taken into account.

A great uncertainty in models for structure formation is the extent and nature of reionization. Fortunately, this uncertainty is largely not reflected in the CMB anisotropies because of the low optical depth to Thomson scattering at the low redshifts in question. Reionization is known to be essentially complete by $z \sim 5$ from the absence of the GunnPeterson effect in quasar absorption spectra (Gunn \& Peterson 1965). Significant reionization before $z \sim 50$ will be ruled out once the tentative detections of the CMB acoustic peaks at present are confirmed (Scott, Silk, \& White 1995). It should be possible to deduce the reionization redshift $z_{i}$ through CMB polarization measurements (Zaldarriaga, Spergel, \& Seljak 1997).

Nevertheless, the duration of time spent in a partially ionized state will remain uncertain. Moreover, as emphasized by Kaiser (1984), secondary anisotropies generated by the Doppler effect in linear perturbation theory are suppressed on small scales for geometric reasons (gravitational instability generates potential flows, leading to cancellations between positive and negative Doppler shifts). Higher order effects that are not generally included in the theoretical modeling of CMB anisotropies are likely to be the main source of secondary anisotropies from reioniza-

\footnotetext{
${ }^{1}$ Alfred P. Sloan fellow.

${ }^{2}$ See the MAP homepage at http://map.gsfc.nasa.gov.

${ }^{3}$ See the Planck Surveyor homepage at http://astro.estec.esa.nl/ SA-general/Projects/Planck.
}

tion below the degree scale. Such effects rely on modulating the Doppler effect with spatial variations in the optical depth. Incarnations of this general mechanism include the Vishniac effect from linear density variations (Vishniac 1987), the kinetic Sunyaev-Zeldovich effect from clusters (Sunyaev \& Zeldovich 1970), and the effect considered here: the spatial variation of the ionization fraction.

Reionization commences when the first baryonic objects form stars or quasars that convert a part of the nuclear or gravitational energy into UV photons. Each such source then blows out an ionization sphere around it. Before these regions overlap, there is a period when the universe is ionized in patches. The extent of this period and the time evolution of the size and number density of these patches depend on the nature of the ionizing engines in the first baryonic objects. Theories of reionization do not give robust constraints (see Tegmark, Silk, \& Blanchard 1994; Rees 1996; Aghanim et al. 1996; Loeb 1997; Haiman \& Loeb 1997; Silk \& Rees 1998; Haiman \& Loeb 1998).

We therefore take a phenomenological approach to studying the effects of patchy reionization on the CMB. We introduce a simple but illustrative three-parameter model for the reionization process based on the redshift of its onset $z_{i}$, the duration before completion $\delta z$, and the typical comoving size of the patches $R$. It is then straightforward to calculate the CMB anisotropies generated by the patchiness of the ionization degree of the intergalactic medium.

We find that only the most extreme models of reionization can produce degree-scale anisotropies that are observable in the power spectrum given the cosmic variance limitations. A large signal on degree scales requires early ionization, $z_{i} \gtrsim 30$, long duration, $\delta z \sim z_{i}$, and ionization in very large patches, $R \gtrsim 30 \mathrm{Mpc}$. Thus the patchiness of reionization is unlikely to affect cosmological parameter estimation from the acoustic peaks in the CMB (Jungman et al. 1996; Zaldarriaga et al. 1997; Bond, Efstathiou, \& Tegmark 1997).

On the other hand, the patchy reionization signal on the subarcminute scale can, in principle, surpass both the primary and the secondary Vishniac signals. These may be 
detectable by the Planck Surveyor and upcoming radio interferometry measurements (Partridge et al. 1997), if point sources can be removed at the $\Delta T / T \sim 10^{-6}$ level.

An explicit expression for the CMB anisotropies power spectrum generated in a universe reionized in patches is given in $\S 2.1$. A simple order of magnitude estimate of the anisotropy from patches is given in $\S 2.2$. In $\S 2.3$ we give a rigorous definition of our three-parameter reionization model and calculate the patchy part of the power spectrum. We discuss illustrative examples in $\S 2.4$.

\section{CMB POWER SPECTRUM}

\subsection{Explicit Expression}

Temperature perturbations $\Delta \equiv \delta T / T$ are generated by Doppler shifts from Thomson scattering. For small optical depths,

$$
\Delta=-\int d l \cdot v \sigma_{\mathrm{T}} n x_{e} .
$$

All quantities here are in physical units. The integral is along the line of site, $v$ is the peculiar velocity of matter, $c=1, \sigma_{\mathrm{T}}$ is the Thomson cross section, $n$ is the number density of free and bound electrons, and $x_{e}$ is the local ionization fraction.

To evaluate equation (1) explicitly, one must specify the cosmological model. For simplicity, we take a universe with critical density in matter throughout; we describe the generalization to an arbitrary FRW universe in $\S 2.3$. We furthermore use comoving coordinates $\boldsymbol{x}$ and conformal time $\eta \equiv$ $\int(1+z) d t=(1+z)^{-1 / 2} \eta_{0}$, where $\eta_{0}=2 / H_{0}$ is the present particle horizon. We observe at $\boldsymbol{x}=0$ and conformal time $\eta_{0}$ along the direction of a unit vector $\hat{\gamma}$; light propagation is given by $\boldsymbol{x}=\hat{\gamma}\left(\eta_{0}-\eta\right)$. Equation (1) can be written as

$$
\Delta(\hat{\gamma})=-\tau_{0} \eta_{0}^{3} \int \frac{d \eta}{\eta^{4}} \hat{\gamma} \cdot v\left[\eta, \hat{\gamma}\left(\eta_{0}-\eta\right)\right] x_{e}\left[\eta, \hat{\gamma}\left(\eta_{0}-\eta\right)\right] .
$$

Here $\tau_{0} \equiv \sigma_{\mathrm{T}} n_{0} \eta_{0}$ is the optical depth to Thomson scattering across the present particle horizon.

The scales contributing to the peculiar velocity are still in the linear regime, therefore

$$
v(\eta, x)=\frac{\eta}{\eta_{0}} v(x)
$$

where $v(x)$ is the peculiar velocity today. The final explicit expression for the CMB temperature perturbation generated during reionization is

$$
\Delta(\hat{\gamma})=-\tau_{0} \eta_{0}^{2} \int \frac{d \eta}{\eta^{3}} \hat{\gamma} \cdot v\left[\hat{\gamma}\left(\eta_{0}-\eta\right)\right] x_{e}\left[\eta, \hat{\gamma}\left(\eta_{0}-\eta\right)\right] .
$$

The correlation function of the temperature perturbations is defined as

$$
C(\theta)=\left.\left\langle\Delta\left(\hat{\gamma}_{1}\right) \Delta\left(\hat{\gamma}_{2}\right)\right\rangle\right|_{\hat{\gamma}_{1} \cdot \hat{\gamma}_{2}=\cos \theta} .
$$

With temperature perturbations given by equation (4), this becomes

$$
\begin{aligned}
C(\theta)= & \tau_{0}^{2} \eta_{0}^{4} \int \frac{d \eta_{1}}{\eta_{1}^{3}} \int \frac{d \eta_{2}}{\eta_{2}^{3}} \\
& \times\left\langle\hat{\gamma}_{1} \cdot v\left(x_{1}\right) \hat{\gamma}_{2} \cdot v\left(x_{2}\right) x_{e}\left(\eta_{1}, x_{1}\right) x_{e}\left(\eta_{2}, x_{2}\right)\right\rangle,
\end{aligned}
$$

where we denote $\boldsymbol{x}_{1} \equiv \hat{\gamma}_{1}\left(\eta_{0}-\eta_{1}\right)$ and $\boldsymbol{x}_{2} \equiv \hat{\gamma}_{2}\left(\eta_{0}-\eta_{2}\right)$.

\subsection{Order of Magnitude Estimates}

Consider the following patchy reionization scenario. The universe was reionized in randomly distributed patches with a characteristic comoving size $R$. The patches appeared at random in space and time. Once a reionized patch appears, it moves with matter. The average ionization fraction, that is the filling fraction of fully ionized patches, grows monotonically from $X_{e}=0$ at high redshifts to $X_{e}=$ 1 at low redshifts. We consider late reionization (optical depth to Thomson scattering is small) and small patches (smaller than the characteristic length scale of the peculiar velocity field). We assume that reionization occurred at redshift $z_{i}$, and the patchy phase duration is given by $\delta z$.

The angular scale of the patchy signal is given by the ratio of the size of patches to the distance to them in comoving coordinates, $\theta \sim R /\left(\eta_{0}-\eta_{i}\right)$. Assuming that the patches are uncorrelated, the spectrum of fluctuations should be white noise above this scale, which agrees with the exact result, as we shall see (eq. [18]).

The rms CMB temperature fluctuation $\Delta$ on scales $\theta$ due to the patchiness can be estimated as follows. Since by assumption different patches are independent, $\Delta \sim N^{1 / 2} \Delta_{p}$. Here $N$ is the number of patches on a line of site, and $\Delta_{p}$ is a temperature fluctuation from one patch, $\Delta_{p} \sim \tau_{p} v\left(z_{i}\right)$. Here $v(z)=(1+z)^{-1 / 2} v(0)$ is the rms peculiar velocity at redshift $z$, and $\tau_{p}$ is the optical depth for one patch, $\tau_{p} \sim\left(1+z_{i}\right)^{2} \sigma_{\mathrm{T}} n_{0} R$. The number of patches $N \sim \delta \eta /$ $R \sim\left(1+z_{i}\right)^{-3 / 2} \delta z \eta_{0} / R$. Collecting all the factors, we get the following estimate for the rms anisotropies from patches:

$$
\Delta \sim \tau_{0}\left\langle v^{2}\right\rangle^{1 / 2}\left(R / \eta_{0}\right)^{1 / 2}\left(1+z_{i}\right)^{3 / 4}(\delta z)^{1 / 2},
$$

which again agrees with the exact result (eq. [15], up to a dimensionless multiplier).

\subsection{Power Spectrum}

We can factor the general expression for the temperature correlation in equation (6) as

$$
\begin{aligned}
C(\theta)=\tau_{0}^{2} & \eta_{0}^{4} \int \frac{d \eta_{1}}{\eta_{1}^{3}} \int \frac{d \eta_{2}}{\eta_{2}^{3}} \\
& \times\left\langle\hat{\gamma}_{1} \cdot v\left(x_{1}\right) \hat{\gamma}_{2} \cdot v\left(x_{2}\right)\right\rangle\left\langle x_{e}\left(\eta_{1}, x_{1}\right) x_{e}\left(\eta_{2}, x_{2}\right)\right\rangle .
\end{aligned}
$$

This assumes that $x_{e}$ and $v$ are independent random fields. This is not strictly correct. The ionization fraction $x_{e}$ must be determined by the density perturbation $\delta$, and the density perturbation is not independent of the peculiar velocity (for example, in the linear regime $\delta=-\frac{1}{2} \eta \nabla \cdot v$ ). However, the ionizing radiation is presumably coming from strongly nonlinear objects, where first stars or quasars are lightening up. At high $z$, the length scales where the density is nonlinear are $\ll 10 \mathrm{Mpc}$ comoving, which is much smaller than the length scales contributing to the peculiar velocity. Under the assumption of scale separation, velocity and density (and hence $x_{e}$ ) are indeed independent.

The correlation function for the local ionization fraction $\left\langle x_{e} x_{e}\right\rangle$ is not known. Our model parameterizes the correlation function through the patch size $R$ and a mean (cosmic time-dependent) ionization fraction $X_{e}(\eta)$,

$$
\begin{aligned}
\left\langle x_{e}\left(\eta_{1}, \boldsymbol{x}_{1}\right) x_{e}\left(\eta_{2}, \boldsymbol{x}_{2}\right)\right\rangle=X_{e}\left(\eta_{1}\right) X_{e}\left(\eta_{2}\right) & \\
& +\left[X_{e}\left(\eta_{\min }\right)-X_{e}\left(\eta_{1}\right) X_{e}\left(\eta_{2}\right)\right] e^{-\left[\left(x_{1}-x_{2}\right)^{2} / 2 R^{2}\right]} .
\end{aligned}
$$


Here $\eta_{\min }=\min \left(\eta_{1}, \eta_{2}\right)$. The Gaussian function is chosen for simplicity; it could have been any function of the separation $x$ that equals 1 at $x=0$ and gradually turns to zero at $x>R$. For the mean ionization fraction $X_{e}$, we assume a change from 0 to 1 at a redshift $z_{i}$, with the transition occurring in a redshift interval $\delta z$. We also assume $\delta z \ll z_{i}$ (this is true in all of the models of reionization of which we are aware).

CMB anisotropies generated (and erased) because of the spatially constant part of the correlation function (eq. [9]), which are obviously the same as in the model with a uniform time-dependent reionization. The spatially varying part is responsible for generating new anisotropies; its contribution to erasing the primary anisotropies is negligible. The anisotropy suppression is mainly determined by the total optical depth to Thomson scattering and is insensitive to the small-scale structure of the ionization fraction $x_{e}(\eta, \boldsymbol{x})$.

The CMB correlation function due to the patchy part only is

$$
\begin{aligned}
C^{(p)}(\theta)= & \tau_{0}^{2} \eta_{0}^{4} \int_{0}^{\eta_{0}} \frac{d \eta_{1}}{\eta_{1}^{3}} \int_{0}^{\eta_{0}} \frac{d \eta_{2}}{\eta_{2}^{3}} \\
& \times I_{12}\left\langle\hat{\gamma}_{1} \cdot v\left(\boldsymbol{x}_{1}\right) \hat{\gamma}_{2} \cdot v\left(\boldsymbol{x}_{2}\right)\right\rangle e^{-\left[\left(\boldsymbol{x}_{1}-\boldsymbol{x}_{2}\right)^{2} / 2 R^{2}\right]},
\end{aligned}
$$

where we denote $I_{12} \equiv X_{e}\left(\eta_{\min }\right)-X_{e}\left(\eta_{1}\right) X_{e}\left(\eta_{2}\right)$. The correlation function is nonnegligible only for $\left|x_{1}-x_{2}\right| \lesssim R$. By assumption, $R$ is much smaller then the characteristic scale of the peculiar velocity field. Also, $\left|x_{1}-x_{2}\right| \lesssim R$ requires that the lines of sight $\hat{\gamma}_{1}$ and $\hat{\gamma}_{2}$ be nearly parallel. Then

$$
\left\langle\hat{\gamma}_{1} \cdot v\left(x_{1}\right) \hat{\gamma}_{2} \cdot v\left(x_{2}\right)\right\rangle \approx \frac{1}{3}\left\langle v^{2}\right\rangle,
$$

where $\left\langle v^{2}\right\rangle$ is the mean squared peculiar velocity today. For $z_{i} \gg 1$, the integral (eq. [10]) is dominated by small conformal times, and we have $\left|x_{1}-x_{2}\right|^{2} \approx \theta^{2}\left(\eta_{0}-\eta_{i}\right)^{2}+$ $\left(\eta_{1}-\eta_{2}\right)^{2}$. Then

$$
\begin{aligned}
C^{(p)}(\theta) \approx & \frac{1}{3} \tau_{0}^{2} \eta_{0}^{4}\left\langle v^{2}\right\rangle e^{-\left[\left(\eta_{0}-\eta_{i}\right)^{2} \theta^{2} / 2 R^{2}\right]} \\
& \times \int_{0}^{\infty} \frac{d \eta_{1}}{\eta_{1}^{3}} \int_{0}^{\infty} \frac{d \eta_{2}}{\eta_{2}^{3}} I_{12} e^{-\left[\left(\eta_{2}-\eta_{1}\right)^{2} / 2 R^{2}\right]} .
\end{aligned}
$$

We assume that $\eta_{i} \delta z /\left(1+z_{i}\right) \gg R$ [with $\left.\eta_{i} \equiv \eta\left(z_{i}\right)\right]$ and that during the patchy phase, $z_{i}>z>z_{i}-\delta z$, the ionization fraction $X_{e}$ grows linearly from 0 to 1 such that eventually both hydrogen and helium are fully ionized. Then

$$
C^{(p)}(\theta)=A e^{-\left(\theta^{2} / 2 \theta_{0}^{2}\right)},
$$

where the characteristic angular scale is

$$
\theta_{0}=\frac{R}{\eta_{0}-\eta_{i}}=\frac{R}{\eta_{0}} \frac{\left(1+z_{i}\right)^{1 / 2}}{\left(1+z_{i}\right)^{1 / 2}-1},
$$

and the amplitude is

$$
A=\frac{\sqrt{2 \pi}}{36} \tau_{0}^{2}\left\langle v^{2}\right\rangle \frac{R}{\eta_{0}} \delta z\left(1+z_{i}\right)^{3 / 2} .
$$

Note that a critical matter-dominated universe is assumed in this expression. To generalize this result, replace $\eta_{0}-\eta_{i}$ with the comoving angular diameter distance in equation (14) and a factor of $\left(1+z_{i}\right)$ in equation (15) with the appropriate velocity growth factor.
The power spectrum is given by the spherical harmonics decomposition

$$
C_{l}^{(p)}=2 \pi \int d \cos \theta P_{l}(\cos \theta) w_{p}(\theta) .
$$

For $l \gg 1$,

$$
C_{l}^{(p)} \approx 2 \pi \int_{0}^{\infty} \theta d \theta J_{0}(l \theta) w_{p}(\theta)=2 \pi A \theta_{0}^{2} e^{-\left(\theta_{0} 2 l 2 / 2\right)} .
$$

The power per octave is

$$
\frac{l^{2} C_{l}^{(p)}}{2 \pi}=A l^{2} \theta_{0}^{2} e^{-\theta_{0}^{2} l^{2} / 2} .
$$

The anisotropy power reaches the maximal value

$$
\left(\frac{l^{2} C_{l}^{(p)}}{2 \pi}\right)_{\max }=\frac{\sqrt{2 \pi}}{18 e} \tau_{0}^{2}\left\langle v^{2}\right\rangle \frac{R}{\eta_{0}} \delta z\left(1+z_{i}\right)^{3 / 2}
$$

at

$$
l_{\max }=\frac{\sqrt{2} \eta_{0}}{R}\left[1-\left(1+z_{i}\right)^{-1 / 2}\right]
$$

\subsection{Discussion}

The signal from patchy reionization in our model depends on four quantities: the rms peculiar velocity $\left\langle v^{2}\right\rangle^{1 / 2}$ today, the redshift of reionization $z_{i}$, its duration $\delta z$, and the characteristic comoving size of the patches $R$. The structure formation model specifies the power spectrum of fluctuations, which in turn tells us the rms peculiar velocity. Let us now consider the patchy reionized signal in the context of a specific model for structure formation.

For illustrative purposes, let us consider a cold dark matter model with $h=0.5, \Omega_{b}=0.1$, and a scale-invariant $n=1$ spectrum of initial fluctuations. Normalizing the spectrum to the COBE detection via the fitting formulae of Bunn \& White (1997; their eqs. [17]-[20]) and employing the analytic fit to the transfer function of Eisenstein \& $\mathrm{Hu}$ (1998; their eqs. [15]-[24]), we find an rms velocity of $\left\langle v^{2}\right\rangle^{1 / 2}=3.9 \times 10^{-3}$. With the present optical depth of $\tau_{0}=0.122 \Omega_{b} h=0.0061$, we have a maximal anisotropy of

$$
\left(\frac{l^{2} C_{l}}{2 \pi}\right)_{\max }=2.41 \times 10^{-15} \frac{R}{\mathrm{Mpc}} \delta z\left(1+z_{i}\right)^{3 / 2}
$$

at

$$
l_{\max }=\frac{16958}{R / \mathrm{Mpc}}\left[1-\left(1+z_{i}\right)^{-1 / 2}\right] .
$$

The power spectrum of the model in principle also tells us the remaining parameters of the ionization: its redshift $z_{i}$, duration $\delta z$, and typical patch size $R$. Unfortunately, these quantities depend on details of the cooling and fragmenting of the first baryonic objects to form the ionizing engines. We therefore consider $5 \lesssim z_{i} \lesssim 50$, which spans the range of estimates in the literature (Tegmark et al. 1994; Rees 1996; Aghanim et al. 1996; Loeb 1997; Haiman \& Loeb 1997; Silk \& Rees 1998; Haiman \& Loeb 1998). Reionization, once it commences, is generally completed in a short time, compared with the expansion time at that epoch $\delta z /$ $\left(1+z_{i}\right)<1$, by the coalescence of patches that are small compared with the horizon at the time $R / \eta_{i} \ll 1$. Again, the exact relations depend on the efficiency with which the first objects form and create ionizing radiation (see, e.g., Tegmark et al. 1994). 
Let us consider an extreme example of $z_{i}=10, \delta z=3$, and $R=20$ Mpc. Then the maximal power is $\approx 5.3 \times 10^{-12}$ at $l \approx 590$, and the primary signal at these scales is $\approx 3 \times 10^{-10}$ : the contribution of the patchy reionization is small in comparison (see Fig. 1). However, in light of the high-precision measurements expected from the MAP and Planck satellites, such a signal is not necessarily negligible. The ultimate limit of detectability through power spectrum measurements is provided by so-called cosmic variance. This arises because we can only measure $2 \ell+1$ realizations of any given multipole such that power spectrum estimates will vary by

$$
\delta C_{\ell}=\sqrt{\frac{2}{2 \ell+1}} C_{\ell}^{\text {(primary) }} .
$$

Detection of a broad feature such as that from patchy reionization is assisted in that we may reduce the cosmic variance by averaging over many $\ell$ 's. We show an example of this averaging in Figure 1 (lower left boxes). In this model, the patchy reionization signal can be detected at the several $\sigma$ level if cosmic variance was the main source of uncertainty. Of course, a realistic experiment also has noise and systematic errors. We also show the noise error contributions expected from the MAP experiment in Figure 1.

An important additional source of uncertainty is provided by other unknown aspects of the model. Indeed, it is hoped that the CMB power spectrum can be used to measure fundamental cosmological parameters to high precision. Excess variance from patchy reionization can, in principle, cause problems for cosmological parameter estimation from the CMB if not included in the model. It would remain undetected and produce parameter misestimates if its signal can be accurately mimicked by variations in the other parameters. Fortunately, the angular signature we find here, $\ell^{2}$ white noise until some cutoff due to the patch size, does not resemble the signature of other cosmological parameters that alter the positions and amplitudes of the acoustic peaks (see Bond et al. 1997; Zaldarriaga et al. 1997). Coupled with the small amplitude of the effect on the 10 arcminute to degree scale for even this extreme model, it

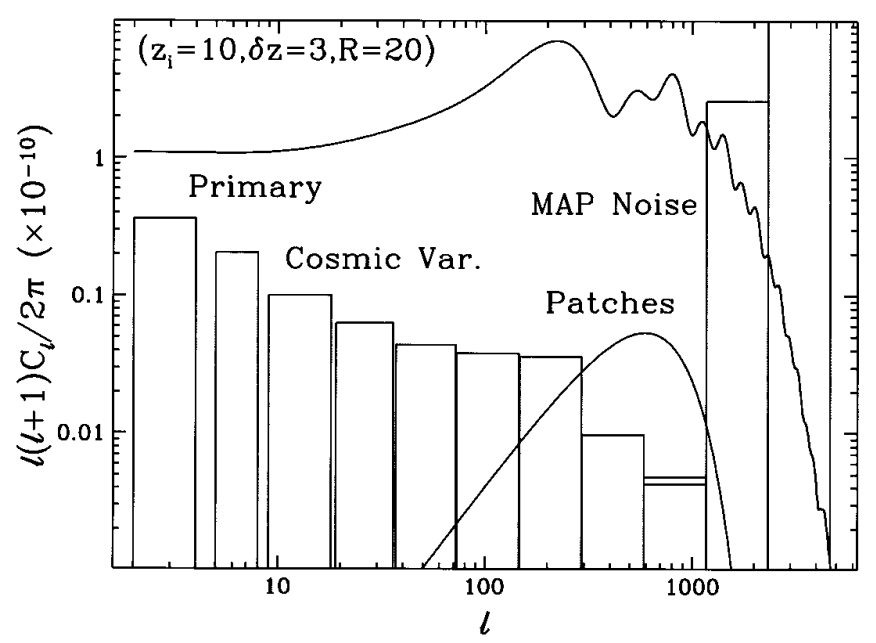

FIG. 1.-CMB anisotropy power spectra in a cold dark matter model with extreme patchiness. Shown here are the primary anisotropy and the patchy reionization anisotropy, eq. (18) with $z_{i}=10, \delta z=3$, and $R=20$ $\mathrm{Mpc}$. These signals are compared with the cosmic variance of the primary anisotropy and the noise of the MAP satellite (in logarithmic bins). is unlikely that patchy reionization will significantly affect parameter estimation through the CMB.

We have called the $\left(z_{i}=10, \delta z=3, R=20\right)$ model extreme because of the size of patches; the reionization redshift and duration would be considered reasonable by a number of theories. For example, the early quasar model of Haiman \& Loeb (1998) does predict $z_{i} \sim 10$ and $\delta z \sim 3$. However, their "medium quasar" emits only $\sim 10^{67}$ ionizing photons during its lifetime. These photons cannot ionize a bubble larger than $R \sim 1 \mathrm{Mpc}$ comoving.

Perhaps more interesting is the case where reionization takes place at a higher redshift with, for example, $z_{i}=30$, $\delta z=5$, and $R=3 \mathrm{Mpc}$. The reduction in the patch size causes the signature to move to smaller angles, where the primary signal is negligible because of dissipational effects at recombination. The increase in the optical depth at this higher redshift is counterbalanced by the reduction in the rms fluctuation due to the number of patches along the line of site such that the amplitude of the signal increases only moderately. Here the maximal power is $\approx 6.2 \times 10^{-12}$ at $l \approx 4650$ (see Fig. 2). Patchy reionization effects exceed the Vishniac signal at these scales $\left(\approx 3 \times 10^{-12}\right)$, which is believed to be the leading other source of secondary anisotropies (Hu \& White 1996).

Although the morphology and amplitude of the patchy reionization and Vishniac signals are similar, the Vishniac effect is fully specified by the ionization redshift and the spectrum of initial fluctuations and hence may be removed once these are determined from parameter estimation at larger angular scales. Likewise, since the rms peculiar velocity $\langle v\rangle$ and the ionization redshift $z_{i}$ will be specified by the large-scale observations, the amplitude of the signal can be used to estimate the duration of reionization $\delta z$ and its angular location the typical comoving size of the bubbles $R$.

In summary, the patchiness of reionization leaves a potentially observable imprint on the CMB power spectrum but one that is unlikely to affect cosmological parameter estimation from the acoustic peaks in the CMB. We show how the signature scales with the gross properties of reionization: its redshift, duration, and typical patch size.

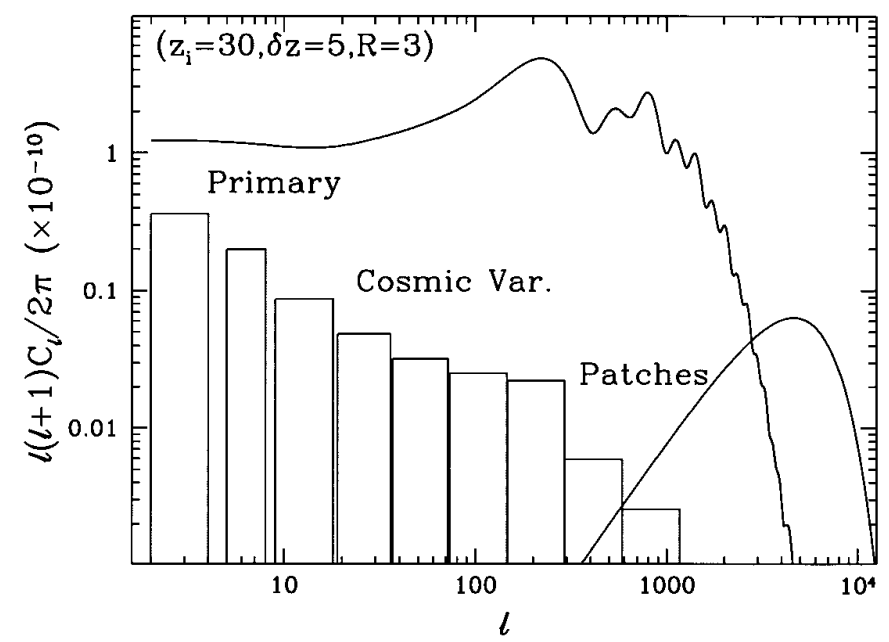

FIG. 2.-CMB anisotropy power spectra in a cold dark matter model with early reionization. Shown here are the primary anisotropy suppressed by rescattering and the patchy reionization anisotropy, eq. (18) with $z_{i}=$ $30, \delta z=5$, and $R=3 \mathrm{Mpc}$. These signals are compared with the cosmic variance of the primary anisotropy achievable by an ideal experiment in the absence of galactic and extragalactic foregrounds. 
Observational detection of this signature would provide useful constraints on the presently highly uncertain reionization scenarios but will likely require experiments with angular resolution of an arcminute or better and foreground subtraction at better than the $\delta T / T \sim 10^{-6}$ level.
We thank R. Juszkiewicz, A. Liddle, M. Tegmark, and M. White for discussions. This work was supported by NSF PHY-9513835. W. H. was also supported by the W. M. Keck Foundation.

\section{REFERENCES}

Aghanim, N., Desert, F. X., Puget, J. L., \& Gispert, R. 1996, A\&A, 311, 1

Kaiser, N. 1984, ApJ, 282, 374

Bond, J. R., Efstathiou, G., \& Tegmark, M. 1997, MNRAS, 291, L33

Loeb, A. 1997, preprint (astro-ph/9704290)

Bunn, E. F., \& White, M. 1997, ApJ, 480, 6

Eisenstein, D. J., \& Hu, W. 1998, ApJ, 511, in press

Gunn, J. E., \& Peterson, B. A. 1965, ApJ, 142, 1633

Haiman, Z., \& Loeb, A. 1997, ApJ, 483, 21 .1998, ApJ, 503, 505

Hu, W., Scott, D., Sugiyama, N., \& White, M. 1995, Phys. Rev. D, 52, 5498

Hu, W., \& White, M. 1996, A\&A, 315, 33

Jungman, G., Kamionkowski, M., Kosowsky, A., \& Spergel, D. N. 1996, Partridge, B., et al. 1997, ApJ, 483, 38

Rees, M. J. 1996, preprint (astro-ph/9608196)

Scott, D., Silk, J., \& White, M. 1995, Science, 268, 829

Silk, J., \& Rees, M. J. 1998, A\&A, 331, L1

Sunyaev, R. A., \& Zeldovich, Ya. B. 1970, Ap\&SS, 7, 3

Tegmark, M., Silk, J., \& Blanchard, A. 1994, ApJ, 420, 484

Vishniac, E. T. 1987, ApJ, 322, 597

Phys. Rev. D, 54, 1332 